\title{
Effects of changes of ischaemic region conductivity in the heart: A 1D model
}

\author{
Peter R. Johnston ${ }^{1}$
}

30 June 2012

\begin{abstract}
The effects of changes in cardiac tissue conductivity within a region of cardiac ischaemia are studied via a simple one dimensional approximation to the full three dimensional bidomain equations governing the electric potential within the heart. This simple model indicates that a greater reduction in intracellular conductivity than in extracellular conductivity leads to an increase in heart surface potentials and vice-versa. It is demonstrated that this result is in accord with observations from the numerical solution of the full three dimensional bidomain equation.
\end{abstract}

\section{Introduction}

The tissue which comprises the human heart is a complex structure of layers of fibres made up of cardiac cells. To model the electric potential distribution in the heart on a continuum level, the bidomain equations were developed [11, 7]. These essentially assume that the intracellular spaces inside the cardiac cells coincide spatially with extracellular spaces, which are outside the cells, but inside the heart wall, and that these two spaces have different electrical properties.

Ischaemia (a result of lack of oxygen in the cardiac cells) induces a change in the resting and plateau transmembrane potentials of the affected cell [9]. The condition also changes the conductivity values of the tissue in the region of the affected cells [9]. This study will investigate how changes in the conductivity values affect the heart surface potential via a simple one dimensional model of the cardiac tissue.

To model the electric field during the ST segment (the plateau potential phase of the cardiac cycle), the bidomain equations are simplified to 
the so-called passive bidomain equation $[2,3]$. This is a Poisson equation governing the potential in the extracellular space with the right hand side of the equation being the generalised Laplacian of the transmembrane potential distribution (the difference between intracellular and extracellular potentials across the cell membrane).

Solving the passive bidomain equation in three dimensions can be a demanding computational task, in terms of code development as well as runtime and memory considerations. Also, solving three dimensional problems numerically may overlook the many subtle interactions which occur. Hence, this paper will present an analysis which approximates the full three dimensional situation by reducing the governing equations to one dimension across the heart wall. The simplified model will be used to study the effect of reduced conductivity in the ischaemic region. Comparisons will be based on changes in the extracellular potential at the origin, which is the analogue of the heart surface potential in a one dimensional sense.

\section{Three Dimensional Formulation}

It is common to study the electric field arising from cardiac ischaemia in a rectangular block of cardiac tissue attached to an infinite blood mass $[6,5$, 4]. The passive bidomain equation $[2,3]$ governs the extracellular electric potential, $\phi_{e}$, during the ST segment, and is given by

$$
\nabla \cdot\left(\mathbf{M}_{i}+\mathbf{M}_{e}\right) \nabla \phi_{e}=-\nabla \cdot \mathbf{M}_{i} \nabla \phi_{m}
$$

where $\phi_{m}$ represents the transmembrane potential distribution in the tissue and $\nabla$ is the usual gradient operator in a $3 \mathrm{D}$ rectilinear coordinate system. The quantities $\mathbf{M}_{i}$ and $\mathbf{M}_{e}$ represent conductivity tensors in the intra- and extracellular spaces, respectively. Also, the potential in the blood, $\phi_{b}$, is governed by Laplace's equation

$$
\nabla^{2} \phi_{b}=0
$$

It will be assumed that the rectangular block of cardiac tissue has fibres on the epicardial (outer heart wall) surface parallel to the $x$-axis. The origin of the $(x, y, z)$ coordinate system is placed in the centre of the tissue block with the epicardial surface represented by the $x-y$ plane (as shown in Figure 1 ). It will be further assumed that the tissue is of thickness $z_{0}$ and is attached to a blood mass, at the endocardial (inner heart wall) surface at $z=z_{0}$, which is of finite size in the $z$-direction such that it is much larger than the thickness of the cardiac tissue. 


\begin{tabular}{l|ll}
\hline Tissue & & \\
& & \\
Blood & & \\
& & \\
& &
\end{tabular}

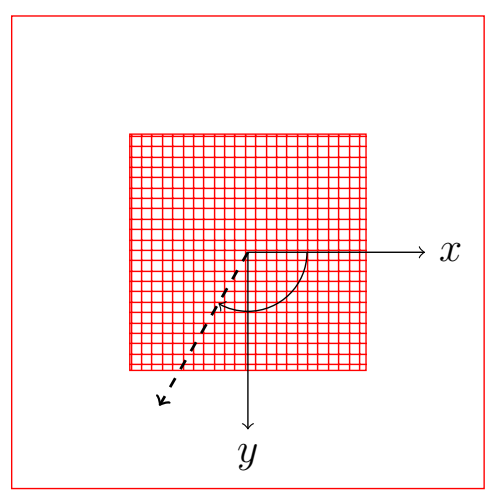

Figure 1: A schematic view of the tissue-blood model used in this study. The left panel is a cross-section view of the tissue $\left(0 \leq z \leq z_{0}\right)$ and the blood $\left(z>z_{0}\right)$ with the positive $z$ axis pointing downwards. The hashed region indicates ischaemic tissue. The right panel is a plan view of the model, looking from the $-z$ direction, again with the hashed region indicating the ischaemic tissue. The dashed arrow indicates the fibre direction on the endocardium (at $z=z_{0}$ ) when the fibre direction on the epicardium (at $z=0)$ points along the $x$-axis.

Placed at the centre of the block of tissue is a region of partial thickness ischaemia extending from the endocardium towards the epicardium. This region of ischaemia is parallelepipedal in shape with sides aligned with the $x$ and $y$ coordinate axes (Figure 1 ).

The conductivity tensors $\mathbf{M}_{i}$ and $\mathbf{M}_{e}$ vary spatially due to the rotation of cardiac fibres from sheet to sheet, with the fibres being parallel within a sheet. For the rectilinear geometry, the conductivity tensors have the form

$$
\mathbf{M}_{k}(x, y, z)=\left(\begin{array}{ccc}
\left(g_{k l}-g_{k t}\right) c^{2}+g_{k t} & \left(g_{k l}-g_{k t}\right) c s & 0 \\
\left(g_{k l}-g_{k t}\right) c s & \left(g_{k l}-g_{k t}\right) s^{2}+g_{k t} & 0 \\
0 & 0 & g_{k n}
\end{array}\right)
$$

where $g_{k l}, g_{k t}$ and $g_{k n}$ are the conductivity values, assumed constant, in the longitudinal, transverse and normal directions to the fibre sheets, respectively, $k=i$ or $e$ (for intracellular and extracellular), $c=\cos (2 \pi z / 3)$ and $s=\sin (2 \pi z / 3)$ to allow for fibre rotation. However, it is possible for the conductivity values to vary spatially as well. In particular, it has been observed that conductivity in ischaemic tissue is less than in normal tissue [9].

The typical boundary conditions associated with the model are that the 
epicardial surface $(z=0)$ is insulated, that is,

$$
\left.\frac{\partial \phi_{e}}{\partial z}\right|_{z=0}=0
$$

It is also assumed that there is continuity of potential and current across the tissue-blood interface

$$
\text { at } z=z_{0}, \quad \phi_{e}=\phi_{b} \text { and } g_{e n} \frac{\partial \phi_{e}}{\partial z}=g_{b} \frac{\partial \phi_{b}}{\partial z}
$$

where $g_{b}$ is the conductivity of blood. It is further assumed that $\phi_{b} \rightarrow 0$ as $z \rightarrow \infty$. The remaining four surfaces of the model geometry are assumed to be insulated.

The transmembrane potential distribution $\phi_{m}$ defines the ischaemic region. If the tissue in the block was everywhere healthy, then $\phi_{m} \equiv 0$. However, in the presence of ischaemic tissue, $\phi_{m}$ is approximately $-30 \mathrm{mV}$ in the ischaemic region with a smooth transition to zero in the normal tissue. This transition is usually represented by a function which is a product of three sigmoidal functions $[11,6]$.

\section{One Dimensional Formulation}

One method to reduce the passive bidomain equation to one dimension is to assume that current within the cardiac tissue can flow only in the $z$-direction, that is, assume that $g_{i l}, g_{i t}, g_{e l}$ and $g_{e t}$ are all zero. The equation governing the electric potential in the one dimensional tissue is then

$$
\frac{d}{d z}\left(\left(g_{i n}+g_{e n}\right) \frac{d \phi_{e}}{d z}\right)=-\frac{d}{d z}\left(g_{i n} \frac{d \phi_{m}}{d z}\right)
$$

where $\phi_{e}$ and $\phi_{m}$ are now simply functions of $z$ only, and the conductivities are given by $g_{i n}(z)$ and $g_{e n}(z)$, respectively. Similarly, the potential in the blood reduces from equation (2) to

$$
\frac{d^{2} \phi_{b}}{d z^{2}}=0
$$

The boundary and interface conditions (3) and (4), respectively, still hold as does the condition that $\phi_{b} \rightarrow 0$ as $z \rightarrow \infty$.

For the one dimensional case, applying this last condition to equation (6) implies that $\phi_{b}(z) \equiv 0$. Hence, at $z=z_{0}$, the one dimensional boundary condition for $\phi_{e}$ is that $\phi_{e}\left(z_{0}\right)=0$. 
Assume that the function $\phi_{m}(z)$ can be written in the form

$$
\phi_{m}(z)=\Delta \phi \sigma(z)
$$

where $\Delta \phi$ is the transmembrane potential in the ischaemic region and $\sigma(z)$ is a sigmoidal function which is twice differentiable and satisfies the conditions that $\sigma(0)=\sigma^{\prime}(0)=\sigma^{\prime}\left(z_{0}\right)=0$ and $\sigma\left(z_{0}\right)=1$. This choice of functional form for $\phi_{m}(z)$ allows the transmembrane potential distribution to vary smoothly from its value in the ischaemic region $(\Delta \phi)$ to its normal value in the healthy tissue (zero).

To include the spatial variation in conductivity, assume that

$$
g_{k n}=G_{k H}+\left(G_{k I}-G_{k H}\right) \sigma(z)
$$

where $k=i$ or $e$ and $G_{k H}$ and $G_{k I}$ are the healthy and ischaemic values of the tissue conductivities. It is reasonable to assume that the transition from normal to ischaemic conductivity values follows the same functional form as the change in transmembrane potential.

\section{Dimensionless Formulation}

To render the problem dimensionless, introduce the dimensionless variables $\eta=z / z_{0}$ and $\Phi_{e}(\eta)=\phi_{e}(z) / \Delta \phi$ to obtain the governing equation

$$
\frac{d}{d \eta}\left(\left(g_{i H}+g_{e H}\right) \frac{d \Phi_{e}}{d \eta}\right)=-\frac{d}{d \eta}\left(g_{i H} \frac{d \sigma(\eta)}{d \eta}\right)
$$

(as $\left.\phi_{m}(z) / \Delta \phi=\sigma(\eta)\right)$ and boundary condition

$$
\left.\frac{d \Phi_{e}}{d \eta}\right|_{\eta=0}=0, \quad \Phi_{e}(1)=0 .
$$

Here

$$
g_{i H}(\eta):=\frac{g_{i n}}{G_{i H}}=1+\left(\frac{G_{i I}}{G_{i H}}-1\right) \sigma(\eta)=1-\left(1-\epsilon_{i}\right) \sigma(\eta)
$$

where $\epsilon_{i}=G_{i I} / G_{i H}$ represents the fractional change in intracellular conductivity. Also,

$$
\begin{aligned}
g_{e H}(\eta) & :=\frac{g_{e n}}{G_{e H}}=\frac{G_{e H}}{G_{i H}}+\left(\frac{G_{e I}}{G_{i H}}-\frac{G_{e H}}{G_{i H}}\right) \sigma(\eta) \\
& =\frac{G_{e H}}{G_{i H}}+\left(\frac{G_{e I}}{G_{e H}} \frac{G_{e H}}{G_{i H}}-\frac{G_{e H}}{G_{i H}}\right) \sigma(\eta)=\beta\left[1-\left(1-\epsilon_{e}\right) \sigma(\eta)\right]
\end{aligned}
$$


where $\epsilon_{e}=G_{e I} / G_{e H}$ represents the fractional change in extracellular conductivity and $\beta=G_{e H} / G_{i H}$ is the relative magnitude of extracellular to intracellular conductivity in normal tissue. Hence the final dimensionless formulation is

$$
\begin{aligned}
& \frac{d}{d \eta}\left(\left((1+\beta)-\left[\left(1-\epsilon_{i}\right)+\beta\left(1-\epsilon_{e}\right)\right] \sigma(\eta)\right) \frac{d \Phi_{e}}{d \eta}\right) \\
& =-\frac{d}{d \eta}\left(\left[1-\left(1-\epsilon_{i}\right) \sigma(\eta)\right] \frac{d \sigma(\eta)}{d \eta}\right)
\end{aligned}
$$

subject to the boundary conditions (8).

\section{Solving the Governing Equation}

As a baseline case, consider the situation where there is no change in ischaemic region conductivity, that is, $\epsilon_{i}=\epsilon_{e}=1$. The governing differential equation (11) then reduces to

$$
(1+\beta) \frac{d^{2} \Phi_{e}}{d \eta^{2}}=-\frac{d^{2} \sigma(\eta)}{d \eta^{2}}
$$

It can be shown easily that the solution of this differential equation subject to the boundary conditions (8) is

$$
\Phi_{e}(\eta)=\frac{1}{1+\beta}(1-\sigma(\eta))
$$

In particular, it follows that the "body surface" potential, $\phi_{e}^{(u)}$, is

$$
\phi_{e}^{(u)}:=\Phi_{e}(0)=\frac{1}{1+\beta}
$$

To solve the more general equation (11), integrate both sides with respect to $\eta$ and use the fact that $\frac{d \Phi_{e}}{d \eta}=\frac{d \sigma}{d \eta}=0$ at $\eta=0$, giving

$$
\left((1+\beta)-\left[\left(1-\epsilon_{i}\right)+\beta\left(1-\epsilon_{e}\right)\right] \sigma(\eta)\right) \frac{d \Phi_{e}}{d \eta}=-\left[1-\left(1-\epsilon_{i}\right) \sigma(\eta)\right] \frac{d \sigma(\eta)}{d \eta}
$$

Following some rearrangement, further integration yields

$$
\Phi_{e}(\eta)=-\int \frac{1-\left(1-\epsilon_{i}\right) \sigma}{(1+\beta)-\left[\left(1-\epsilon_{i}\right)+\beta\left(1-\epsilon_{e}\right)\right] \sigma} d \sigma
$$


Then, using [10, equation 14.111] and the facts that $\Phi_{e}(1)=0$ and $\sigma(1)=1$, gives

$$
\begin{aligned}
\Phi_{e}(\eta)= & \frac{1-\epsilon_{i}}{\left(1-\epsilon_{i}\right)+\beta\left(1-\epsilon_{e}\right)}(1-\sigma(\eta)) \\
& -\frac{\beta\left(\epsilon_{i}-\epsilon_{e}\right)}{\left[\left(1-\epsilon_{i}\right)+\beta\left(1-\epsilon_{e}\right)\right]^{2}} \ln \left|\frac{\epsilon_{i}+\beta \epsilon_{e}}{(1+\beta)-\left[\left(1-\epsilon_{i}\right)+\beta\left(1-\epsilon_{e}\right)\right] \sigma(\eta)}\right|
\end{aligned}
$$

It immediately follows that the body surface potential is given by

$$
\Phi_{e}(0)=\frac{1-\epsilon_{i}}{\left(1-\epsilon_{i}\right)+\beta\left(1-\epsilon_{e}\right)}-\frac{\beta\left(\epsilon_{i}-\epsilon_{e}\right)}{\left[\left(1-\epsilon_{i}\right)+\beta\left(1-\epsilon_{e}\right)\right]^{2}} \ln \left|\frac{\epsilon_{i}+\beta \epsilon_{e}}{1+\beta}\right|
$$

as $\sigma(0)=0$.

\section{Observations from the Model}

The first observation to make from the general solution (equation (13)) is that when $\epsilon_{i}=\epsilon_{e}$, that is, the intra- and extracellular conductivities in the ischaemic region region are reduced by the same amount,

$$
\Phi_{e}(0)=\frac{1}{1+\beta}=\phi_{e}^{(u)}
$$

which is the same value as when there is no change in the ischaemic region conductivities (equation (12)).

In order to study the effect of different relative changes in the ischaemic conductivities, define the function

$$
f\left(\epsilon_{e}, \epsilon_{i}\right):=\Phi_{e}(0)-\Phi_{e}^{(u)}
$$

From equations (13) and (14), it follows that

$$
f\left(\epsilon_{e}, \epsilon_{i}\right)=\frac{\beta\left(\epsilon_{e}-\epsilon_{i}\right)}{\left[\left(1-\epsilon_{i}\right)+\beta\left(1-\epsilon_{e}\right)\right]^{2}}\left[\frac{\left(1-\epsilon_{i}\right)+\beta\left(1-\epsilon_{e}\right)}{1+\beta}+\ln \left|\frac{\epsilon_{i}+\beta \epsilon_{e}}{1+\beta}\right|\right]
$$

It also now follows that $f(\gamma, \gamma)=0$, for $0 \leq \gamma<1$, with the case for $\gamma=1$ following from Equation (14).

A surface and contour plot of the function $f$ for $0 \leq \epsilon_{i}, \epsilon_{e} \leq 1$ is shown in Figure 2, with $\beta=1$. From the figure it can be seen that if $\epsilon_{e} \lessgtr \epsilon_{i}$ then $f\left(\epsilon_{e}, \epsilon_{i}\right) \gtrless 0$. In fact, these statements are true of all values of $\beta$. 


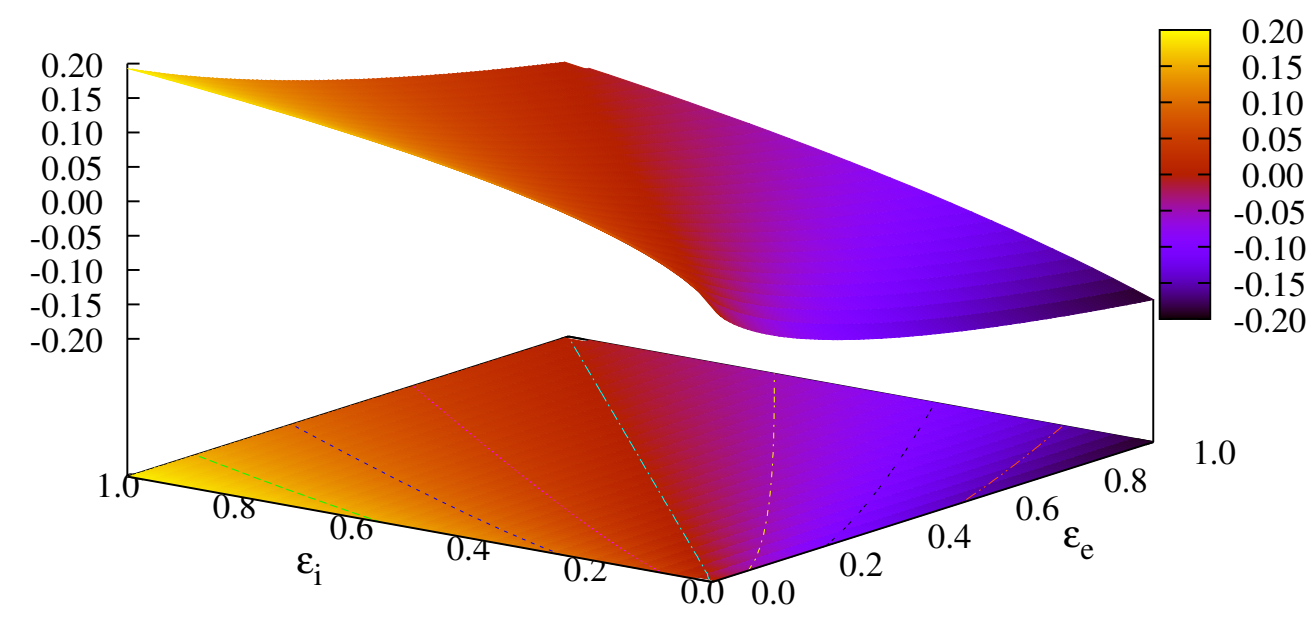

Figure 2: A surface and contour plot of the function $f\left(\epsilon_{e}, \epsilon_{i}\right)$ (Equation (15)) with $\beta=1$.

To prove the assertion, it will be demonstrated that the term in the square brackets in Equation (16) is always negative. Firstly, observe that

$$
\frac{\epsilon_{i}+\beta \epsilon_{e}}{1+\beta}=1-\frac{\left(1-\epsilon_{i}\right)+\beta\left(1-\epsilon_{e}\right)}{1+\beta}
$$

and

$$
0 \leq \frac{\left(1-\epsilon_{i}\right)+\beta\left(1-\epsilon_{e}\right)}{1+\beta}<1
$$

Then, using the Taylor series expansion for $\ln (1+x)$ near $x=0 \quad$ [10, equation (20.17)], it follows that

$$
\begin{aligned}
& \frac{\left(1-\epsilon_{i}\right)+\beta\left(1-\epsilon_{e}\right)}{1+\beta}+\ln \left|\frac{\epsilon_{i}+\beta \epsilon_{e}}{1+\beta}\right|= \\
& -\frac{1}{2}\left[\frac{\left(1-\epsilon_{i}\right)+\beta\left(1-\epsilon_{e}\right)}{1+\beta}\right]^{2}-\frac{1}{3}\left[\frac{\left(1-\epsilon_{i}\right)+\beta\left(1-\epsilon_{e}\right)}{1+\beta}\right]^{3}-\ldots<0 .
\end{aligned}
$$

Hence, if $\epsilon_{e}>\epsilon_{i}$, then $f\left(\epsilon_{e}, \epsilon_{i}\right)<0$ and if $\epsilon_{e}<\epsilon_{i}$, then $f\left(\epsilon_{e}, \epsilon_{i}\right)>0$, regardless of the value of $\beta$.

One further observation is that if $\beta=1$, then $f\left(\epsilon_{e}, \epsilon_{i}\right)=-f\left(\epsilon_{i}, \epsilon_{e}\right)$, which follows from Equation (16) and is indicated by the asymmetry of the contour lines in Figure 2. 


\section{Discussion and Conclusions}

As mentioned above, $f(\gamma, \gamma)=0$ for $0 \leq \gamma \leq 1$. In the context of the electrophysiology problem under consideration, this means that equal reductions in both the intra- and extracellular conductivity values in the ischaemic region do not affect the body surface potential. Further, since $\epsilon_{e}>\epsilon_{i}$ implies there is a larger reduction in the ischaemic intracellular conductivity, then $f\left(\epsilon_{e}, \epsilon_{i}\right)<0$ implies that the body surface potential is lower than that observed for totally healthy tissue. Finally, when the ischaemic extracellular conductivity is reduced by a larger amount $\left(\epsilon_{e}<\epsilon_{i}\right)$, the body surface potential is higher than normally observed.

To check the validity of the simplifying assumptions made in this study, simulations were performed using a finite volume method solution for a three dimensional block of tissue [4]. In the first simulation, the intracellular and extracellular conductivity values in the longitudinal and transverse directions were set to zero and the conductivities in the normal direction were set to 1. This scenario mimics the one dimensional approximation. The resulting surface plot of the function $f\left(\epsilon_{e}, \epsilon_{i}\right)$ for $0 \leq \epsilon_{e}, \epsilon_{i} \leq 1$ corresponds exactly to that shown in Figure 2. This is perhaps expected as the current has been restricted to flowing in only the $z$ direction.

Finally, consider the full three dimensional situation where the six conductivities take the values $g_{i l}=2.63, g_{e l}=2.63, g_{i t}=0.263, g_{e t}=2.45$,

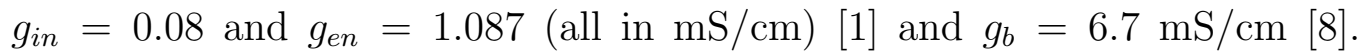
Figure 3 shows a surface plot of the epicardial potential at a point over the centre of the ischaemic region. Here the cardiac fibres rotate through $120^{\circ}$ anticlockwise, from the epicardium to the endocardium, as a function of changes in the normal direction intra- and extracellular conductivities in the ischaemic region. It is interesting to compare Figures 3 and 2. It appears from Figure 3 that the presence of the extra non-zero conductivities swamps the effect of changes to the normal direction conductivities. For example, reducing the ischaemic normal direction intra- and extracellular conductivities by the same amount no longer leaves the epicardial potentials unchanged. In fact, as the conductivities reduce, the epicardial potential increases. However, against this trend, a study of the contour lines on the base of the figure shows that there is still a decrease in epicardial potentials along the line $\epsilon_{e}=1-\epsilon_{i}$. Hence the simple one dimensional model is able to explain one feature of the surface shown in Figure 3. 


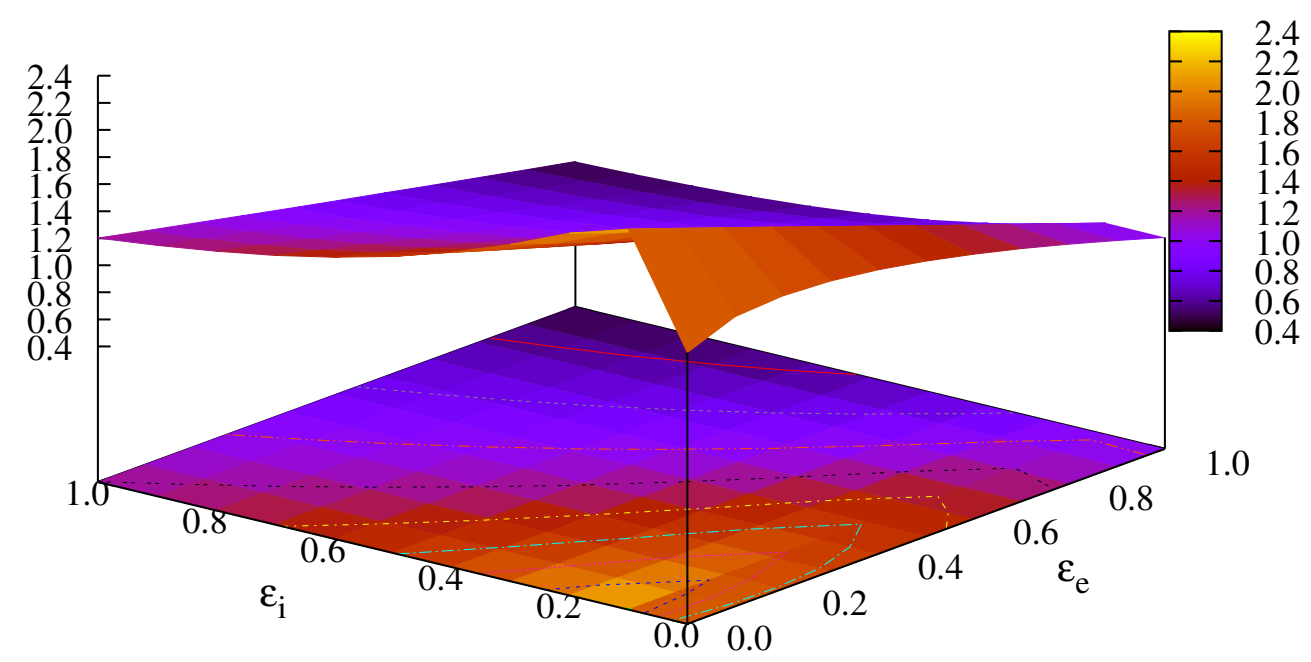

Figure 3: A surface and contour plot of the epicardial potential $\phi_{e}$ above the centre of the ischaemic region for a full three dimensional solution of Equation (1). Physical parameters are specified in the text.

\section{References}

[1] Darren A. Hooks, Mark L. Trew, Bryan J. Caldwell, Gregory B. Sands, Ian J. LeGrice, and Bruce H. Smaill. Laminar arrangement of ventricular myocytes influences electrical behavior of the heart. Circulation Research, 101(10):e103-112-e103-112, 112007.

[2] B Hopenfeld, J. G. Stinstra, and R. S. MacLeod. Mechanism for ST depression associated with contiguous subendocardial ischaemia. Journal of Cardiovascular Electrophysiology, 15:1200-1206, 2004.

[3] Bruce Hopenfeld, Jeroen G. Stinstra, and Rob S. MacLeod. The effect of conductivity on st-segment epicardial potentials arising from subendocardial ischemia. Annals of Biomedical Engineering, 33(6):751-763, 062005.

[4] Peter R. Johnston. A finite volume method solution for the bidomain equations and their application to modelling cardiac ischaemia. Computer Methods in Biomechanics and Biomedical Engineering, 13(2):157-170, 2010.

[5] Peter R. Johnston and David Kilpatrick. The effect of conductivity values on $\mathrm{ST}$ segment shift in subendocardial ischaemia. IEEE 
Transactions on Biomedical Engineering, 50(2):150-158, February 2003.

[6] Peter R. Johnston, David Kilpatrick, and Chuan Yong Li. The importance of anisotropy in modelling ST segment shift in subendocardial ischaemia. IEEE Transactions on Biomedical Engineering, 48(12):1366-1376, December 2001.

[7] W. T. Miller and D. B. Geselowitz. Simulation studies of the electrocardiogram I: The normal heart. Circulation Research, 43:301-315, 1978.

[8] S. Rush, J. A. Abildskov, and R. McFee. Resistivity of body tissues at low frequencies. Circulation Research, 12:40-50, 1963.

[9] Robin M. Shaw and Yoram Rudy. Electrophysiologic effects of acute myocardial ischemia: a theoretical study of altered cell excitability and action potential duration. Cardiovascular Research, 35(2):256-272, 1997.

[10] Murray R. Spiegel. Mathematical Handbook of Formulas and Tables. Schaum's Outline Series. McGraw-Hill, International edition, 1990.

[11] L. Tung. A Bi-domain model for describing ischaemic myocardial D-C potentials. PhD thesis, Massachusetts Institute of Technology, June 1978.

\section{Author address}

1. Peter R. Johnston, School of Biomolecular and Physical Sciences, Griffith University, Nathan, Queensland 4111, Australia.

mailto:p.johnston@griffith.edu.au 